\title{
Représentation graphique facilitant la recherche d'aménagements hydro=électriques rationnels
}

\author{
pạr Robert Blon, Ingéniẹur hydraulicien à la Compagnie générale d'éleclricilé
}

L'étude d'un aménagement hydroélectrique peut consister en la recherche des disposilions permettant de capter l'énergie d'une rivière, entre deux points bien déterminés de son cours. C'est le cas des installations destinces à exploiter l'avantage de conditions locales, hydrographiques ou topographiques, particulièrement favorables. Une déclivité naturelle importante, par exemple, ou un emplacement convenable à l'édification d'un barrage, peuvent inposer, par des raisons techniques et nomique d'énergie, et dont l'étendue, ou la complexilé nécessit un aménagement divisé en plusieurs chutes distincles. L'élud de la répartition rationnelle des différentes chutes sur l'ensemb dı système envisagé, devient alors un problème très délicat, ‘u dans beaucoup de cas, ne peut pas être résolu d'unc façon salisfa sante par les moyens de prospection ordinaires. En effet, malg la quantité et la précision des renseignements qu'on a pu recueill sur les caractéristiques naturelles des rivières à aménago

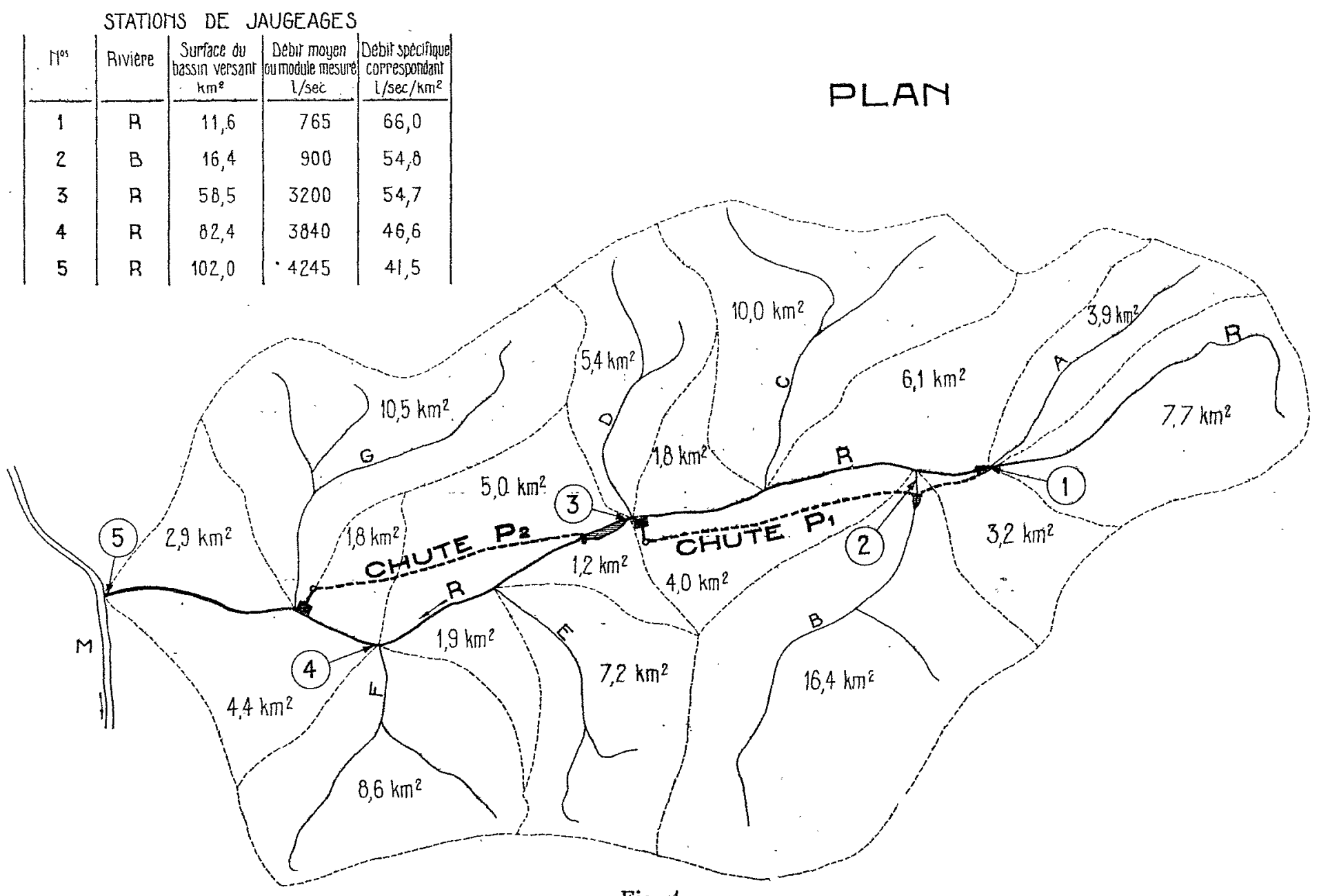

Fig. 1

économiques évidentes, les limites rationnelles d'utilisation d'un cours d'eau. Nous ne parlons ici, bien entendu, que des limites géographiques d'utilisation, l'étude de l'aménagement proprement dit (emplacements et dimensions des ouvrages) reste, dans tous les cas, un problème complexe dont la solution doit satisfaire à un grand nombre de conisidérations d'ordres divers.

La question est toute différente lorsqu'il s'agit non plus d'une installation hydroélectrique isolée, mais de la captation d'un cours d'eau ou d'un système de cours d'eau, présentant, il est inévitable de mener l'étude par tâtonnements, lorsqui ne possède pas un moyen de coordonner les différents élémon qui concourent à domner plus ou moins de valeur producti à une installation ou à une autre.

C'est devant cette difficulté que nous avons cherché à rện senter graphiquement, en une seule figure, l'importance de quantité d'énergie susceptible d'être tirée d'entre deux point quelconques d'un système.

Nous supposons connues les dispositions topographiciues gép rales des cours d'eau à étudier," soit : un plan avec indicat

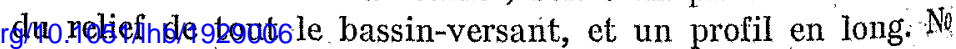




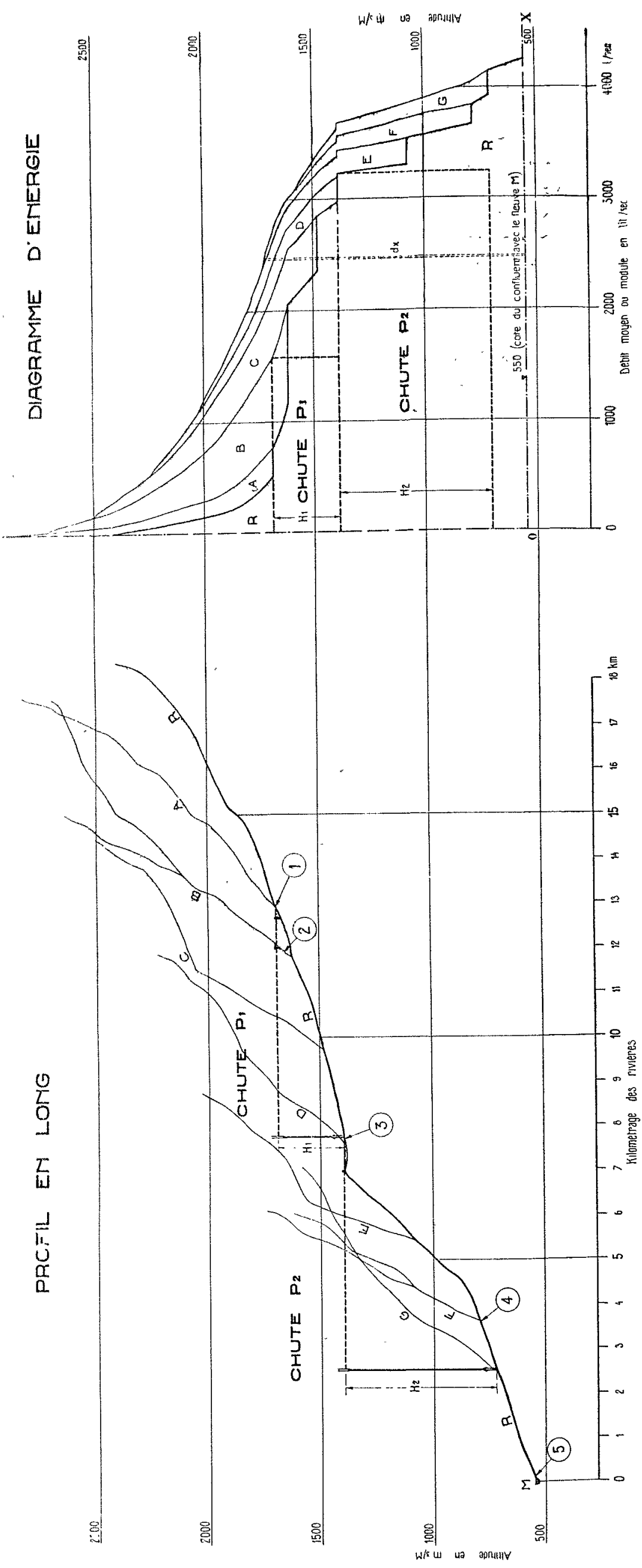

admettons aussi que des observations suffisamment complètes ont été faites sur les débits, pour permettre d'évaluer le régime approximatif en n'importe quel point de l'ensemble. Dans l'exemple que nous donnons ici (fig. 1 : plan ; fig. 2 : profil en long), cinq stations de jaugeages ont été installées aux endroits les plus caractéristiques du bassin versant, et les résultats des observations sont portés sous forme du chiffre de débit moyen ou module relevé à chacune d'elles.

La représentation graphique préconisée (fig. 2) qui est, en réalité, un diagramme d'énergie, est obtenue de la façon suivante :

- les ordonnées sónt les altitudes comptées audessus d'un plan de comparaison $\mathrm{O}-\mathrm{X}$ situé audessous du point le plus bas de l'aménagement à étudier (les altitudes sont ici en projection avec celles du profil en long).

- les abscisses représentent, comptées depuis un axe vertical quelconque $\mathrm{O}-\mathrm{Y}$, le débit annuel moyen ou module correspondant à l'altitude.

En d'autres termes, la longueur totale d'une abscisse d'altitude déterminée représente le débit moyen total passant à travers un plan horizontal situé à cette altitude, et coupant tous les cours d'eau du système étudié. Les débits moyens de chaque cours d'eau sont cumulés pour fournir l'abșcisse totale à une altitude donnée. C'est ainsi que le débit de la rivière principale $R$ est porté le plus près de l'axe $\mathrm{O}-\mathrm{Y}$ (trait fort), et les débits de chaque affluent $(\mathrm{A}, \mathrm{B}, \mathrm{C})$ totalisés, à la suite, sur la même abscisse (traits fins). On voit alors, à l'altitude d'un confluent, le débit moyen de la rivière $\mathrm{R}$ brusquement augmenté du débit de l'affluent.

Les différents points permettant la construction de ces courbes de débits moyens en fonction de l'altitude, sont calculés par interpolation et extrapolation, en partant des résultats obtenus aux différentes stations de jaugeages et en s'aidant des surfaces des bassins versants élémentaires tels qu'ils sont tracés au plan de la figure 1. Les observations pluviométriques, s'il en existe, peuvent être très utiles pour établir la répartition des débits spécifiques moyens sur les bassins versants élémentaires.

Considérons un élément vertical de largeur $d x$ ct de hauteur $y$, limité par l'axe $0-x$ et par la courbe des débits moyens totaux (courbe extrême) de la figure obtenue; la surface de cet élément, $d s=y d x$, représente le produit d'un élément de débit moyen par la hauteur dont il descend avant de traverser le plan $\mathrm{O}-\mathrm{X}$, et, par suite, la puissance qu'il développe pendant cette descente. La surface de la figure comprise entre deux plans d'altitudes $\mathrm{Y}_{1}$ et $\mathrm{Y}_{2}$, a pour valeur

$$
\mathrm{S}=\int_{\mathrm{Y}_{1}}^{\mathrm{Y}_{2}} \mathrm{Y} d x
$$

et représente la puissance moyenne totale développée par les eaux du système pendant leur chute de l'altitude $Y_{R}$ à l'altitude $Y_{1}$. Enfin, la surface totals 
de la figure sensiblement triangulaire limitée par les axes $O-X$, O-Y, et par la courbe extrème des débits moyens, représente la puissance moyenne totale développée par toutes les eaux des rivières considérées, depuis leurs sources respectives, jusqu’à leur passage à travers le plan inférieur $\mathrm{O}-\mathrm{X}$.

On remarque que la puissance moyemne d'une installation hydrólectrique quelconque, peut alors être représentée par un rectangle inscrit dans le diagramme. Le rectangle aura pour largeur le débit moyen disponible (abscisse ̀̀ l'altitude de la prise d'eau), pour hauteur, la hauteur de chute de l'installation, et sa surface sera la puissance moyenne théorique disponible.

L'aménagement d'ensemble le plus productif est alors celui dont la construction graphique etablie ainsi, recouve la plus grande partie de la surface du diagramme.

En s'aidant du plan et du profil en long, il est possible de représenter de cette façon n'importe çuel type d'installation, y compris toutes les combinaisons de traversée d'une vallée dans une antre, de captation d'affluents au passage, et même d'accumulation par pompage.

A titre d'exemple, nous avons figuré ici, en plan, en profil ct sur le diagramme d'énergie les deux chutes $P_{1}$ et $P_{2}$, dont les seules particularités sont que la dérivation de la chute $P_{1}$ capte au passage les eaux de l'affluent $B$, et que la prise d'eau de la chute $P_{2}$ est faite dans un lac naturel.

Cet exemple est purement schématique et ne résulte pas d'une étude. On voit que la puissance moyenne théorique de ces deux installations très simples représente environ $50 \%$ de la puissance moyenne naturelle totale développée par tous les cours d'eau du système, depuis leurs sources jusqu'au confluent du fleuve M. Il aurait été très difficile, sans le secours du diagramme d'énergie, de dégager ce schéma d'aménagement qui, dans ses grandes lignes, fait certainement partie de l'un des plus rationnels.

Une foule d'autres chutes peuvent être encore aménagćes sur le bassin étudié, mais il apparaît nettement que les deux installations $P_{1}$ et $P_{2}$ sont la base de l'aménagement rationnel d'ensemble et que, dans un schéma qui les négligerait, il fau- drait un plus grand nombre d'usines pour obtenir une production d'énergie de même importance.

Un point particulier de l'exemple donné ici, montre l'utilit de l'emploi du diagramme d'énergie : c'est le choix de la limit aval de la chute $P_{2}$ (lieu de restitution des eaux). L'examen th plan et du profil en long seuls peut conduire à préconiser de silut l'usine $\mathrm{P}_{2}$ en amont immédiat du confluent de $\mathrm{F}$ et de reprend en une nouvelle chute $p_{3}$, toutes les eaux disponibles en ce poin (y compris celles de $F$ ) pour ne les restituer qu'au fleuve Or, la représentation graphique de ces deux nouvelles chut $p_{2}$ et $p_{3}$ (non figurées ici), sur le diagramme d'énergie, fait $r$ sortir au premier coup d'oil un accroissement de producti d'environ $25 \%$ (par rapport à la chute $\mathrm{P}_{2}$ du premier schémá tandis que la longueur des canaux d'amenée nécessaires augmentée de $75 \%$ et que le: travaux comportent une pri: d'eau et une usine supplémentaires. D'autre part, le prolong ment de la chute $P_{z}$ jusqu'au fleuve $M$ fait apparaître, de mème façon, un aceroissement de production de $20 \%$ sen ment (rectangle $\mathrm{P}_{2}$ du diagramme d'énergie descendu jusqu l'axe $\mathrm{O}-\mathrm{X})$, tandis que l'allongement du canal d'amence de $75 \%$.

Il est évident que la méthode ne permet pas, à elle seule, déterminer le moyen le plus économique de capler l'éneri d'un ensemble de rivières. Le but à atteindre est rarement dl' tenir le maximum de kWH sous une forme quelconque. nécessité de régulariser les débits naturels, fait que le problè: de recherche des emplacements propices à l'aménagement; réservoirs artificiels, reste entier. De plus, des conditions top graphiques ou géologiques défavorables peuvent s'opposer. mellement à la réalisation d'un schéma qui, par ailleurs, $p:$ sente des avantages productifs indiscutables.

Le diagramme d'énergie permet, lorsqu'on entreprend l'éti d'un aménagement hydraulique complexe, de se diriger, les premiers pas, vers les solutions présentant les meillell qualités théoriques. Par la suite, lorsque des considérali locales ont conduit à modifier le premier schéma théorique en sagé, et que la solution pratique est dégagée, l'emploi du h gramme d'énergie laisse la certitude qu'aucune tranche d'éneri naturelle susceptíble d'être captée avantageusement, n'a i ignorée. 